\title{
ALOCAÇÃO DE PÓLOS ROBUSTA PARA UM MOTOR DE UMA JUNTA ROBÓTICA
}

\author{
VINÍCIUS A. B. DOS SANTOS ${ }^{1}$, MÁRCIA L. M. PRADO ${ }^{2}$, ANFRANSERAI M. DIAS ${ }^{2}$.
}

1. Escola Politécnica, Universidade Federal da Bahia, Rua Aristides Novis, 02, $2^{\circ}$ andar, Federação, Salvador-BA. CEP: 40210-630

E-mail: vinigustobittencourtegmail.com

\section{Laboratório de Sistemas de Controle e Robótica, Departamento de Tecnologia, Universidade Estadual de Feira de Santana, Av. Transnordestina, s/n, Novo Horizonte, Feira de Santana-BA. CEP: 44036- 900 \\ E-mails: marcia.lissandra@gmail.com, anfranseraidecomp.uefs.br}

\begin{abstract}
This article presents the robust pole placement using modal intervals applied to system of joint motor of a robotic manipulator, more specifically in a motor current DC. The technique uses modal interval analysis and PD controller for speed feedback. The aim of applying robust control of dc motor plant to ensure satisfactory performance is within the requirements of the project, even if the system is subject to uncertainties in the parameters of the mathematical model of the plant.
\end{abstract}

Keywords_ Control Systems, Robust Control, Robotic Manipulator, Modal Intervals.

Resumo - Este artigo apresenta a alocação de pólos robusta utilizando intervalos modais aplicado a um sistema de motor de junta de um manipulador robótico, mais especificamente em um motor de corrente contínua (CC). A técnica faz uso da análise intervalar modal e controlador PD por realimentação de velocidade. O objetivo de aplicar controle robusto à planta do motor CC é garantir desempenho satisfatório dentro dos requisitos do projeto, mesmo que o sistema esteja sujeito a incertezas nos parâmetros do modelo matemático da planta.

Palavras-chave— Sistemas de Controle, Controle Robusto, Manipulador Robótico, Intervalos Modais.

\section{Introdução}

Grande parte das indústrias utiliza o controle proporcional-integral-derivativo (PID) em seus sistemas, pelo fato de ser de fácil implantação, possuir simplicidade funcional e facilidade de sua utilização com tecnologia atual (Gutierrez, 2010). Porém, em algumas situações, os controladores PID tradicional podem não apresentar um desempenho satisfatório/adequado (Gutierrez, 2010), por exemplo, nos casos de plantas com fortes não-linearidades e sistemas em que os parâmetros variam com o tempo.

Uma abordagem que pode ter mais aplicabilidade em sistemas em que os seus parâmetros podem variar com o tempo é a técnica de controle robusto. Um controlador é dito robusto quando o sistema a ser controlado esteja sujeito a incertezas nos parâmetros da planta e/ou perturbações externas, e a resposta do sistema ainda se mantém dentro das especificações de projeto (Ackermann, 1993; Barmish, 1994; Battacharyya, Chapellat e Keel, 1995 apud Prado, 2006).

São várias as técnicas existentes de projetos de controladores robustos (Zhou, Doyle e Glover, 1996), porém, normalmente envolvem projetos de controladores no espaço de estados. Técnicas de controladores robustos utilizando controladores PID podem ser mais vantajosas quando aplicadas a algumas indústrias devido a simplicidade do controlador.

Com este trabalho, propõem-se utilizar a metodologia abordada em Prado (2008) para projetar controladores robustos em um sistema eletromecânico como um motor CC. Esta metodologia leva em conta incertezas nos parâmetros da planta, e estas incertezas são consideradas utilizando aritmética intervalar modal, ou seja, fazendo uso de intervalos e de uma representação semântica, ao invés de valores pontuais (fixos).

Neste trabalho, não apenas foram projetados controladores para um manipulador robótico, como também, foram realizadas simulações via software do controlador robusto.

Este artigo está dividido da seguinte forma, na Seção 2 será apresentada uma breve fundamentação teórica, necessária para o desenvolvimento do projeto, como: controlador PD por realimentação de velocidade, aritmética intervalar modal e controle robusto. Na Seção 3 é apresentada a metodologia de projeto desde a modelagem matemática do motor CC até a aplicação da metodologia de controle robusto utilizando aritmética intervalar modal. Na Seção 4 são apresentados os resultados da pesquisa. E por fim, na Seção 5, são descritas as conclusões obtidas com a pesquisa.

\section{Fundamentação Teórica}

\subsection{Controle PD por Realimentação de Velocidade}

Considerando $u(t)$ como sendo o sinal da ação de controle (sinal de saída do bloco controlador) e $e(t)$, 
o sinal de erro, pode-se definir os tipos de controladores de acordo com a relação entre $u(t)$ e $e(t)$. No controlador proporcional: $u(t)=k_{p} e(t)$, no integral: $u(t)=k_{i} \int e(t) d t$; no derivativo: $u(t)=k_{d} d e(t) / d t$; e no PID: $u(t)=k_{p} e(t)+k_{i} \int e(t) d t+k_{d} d e(t) / d t$. Os valores de $k_{p}, k d$, e $k_{i}$ são constantes (Ogata, 2003). O controle proporcional-derivativo (PD) pode ser visualizado na Figura 1.

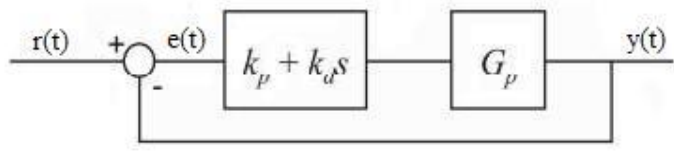

Figura 1 - Diagrama de Bloco do Controle PD

Uma ação de controle derivativa, quando acrescentada a um controlador proporcional, permite que se obtenha um controlador de alta sensibilidade. Uma vantagem em utilizar esta ação de controle é que a mesma responde a uma taxa de variação de erro atuante e pode produzir uma correção significativa antes que o valor do erro atuante se torne muito elevado (Ogata, 2003). No domínio do tempo, o sinal de controle com o termo proporcional-derivativo é dado por:

$$
u(t)=k_{p} e(t)+k_{d} \frac{d e}{d t}
$$

A função de transferência de malha fechada (relação entre entrada e saída no domínio da frequência) (Ogata, 2003) é dada por:

$$
G_{M F}=\frac{G_{p}\left(k_{p}+k_{d} s\right)}{1+G_{p}\left(k_{p}+k_{d} s\right)}
$$

Os zeros do sistema são as raízes do numerador da função de transferência, e os pólos do sistema são as raízes do denominador (Ogata, 2003).

O controle PD produz um zero na função de transferência de malha fechada, podendo causar oscilações na resposta do sistema. Para evitar este comportamento indesejável pode-se utilizar um controle PD por realimentação de velocidade, Figura 2.

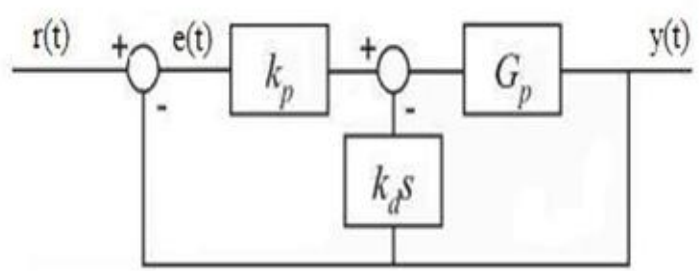

Figura 2 - Diagrama de Bloco do Controle PD com realimentação de Velocidade

A função de transferência de malha fechada do controle PD por realimentação de velocidade é dada por:

$$
G_{M F}=\frac{G_{p} k_{p}}{1+G_{p}\left(k_{p}+k_{d} s\right)}
$$

Para se realizar o projeto do controlador, basta selecionar os ganhos $k_{p}$ e $k_{d}$, de forma que o sistema de malha fechada possua uma saída que satisfaça às especificações de projeto. Isto pode ser obtido através de comparação do sistema a malha fechada desejado com a expressão de $G_{M F}$.

O tempo de acomodação, parâmetro de especificação de desempenho para sistemas de segundaordem, pode ser calculado através da seguinte equação:

$$
t_{s} \cong \frac{4,8}{\xi \omega_{n}}
$$

Vale ressaltar que a equação (4) é utilizada para $\xi=1$ (Ogata, 2003).

\subsection{Aritmética Intervalar Modal}

A análise intervalar modal caracteriza intervalos a partir de números reais com os conjuntos de predicados que os intervalos aceitam ou rejeitam (Prado, 2006).

Pode-se definir um intervalo modal como um par formado por um intervalo clássico (ou seja, um conjunto de números reais) $[x]=\left[x^{-}, x^{+}\right]$e um quantificador $Q[x]$ '.

$$
[x]^{\prime}=\left([x], Q[x]^{\prime}\right) .
$$

O quantificador representa a modalidade de um intervalo, podendo ser: Existencial, existe um $x \in$ $[x]$, com $Q[x]^{\prime}=\mathrm{E}$; e Universal, para todo $x \in[x]$, com $Q[x]^{\prime}=U$.

Sendo que os predicados são condições que podem ser relacionadas a um intervalo, por exemplo,

$$
y=\{x \in[x], \mid x \geq 0\} .
$$

Ou seja, o predicado desta condição é $x \geq 0$. O conjunto dos intervalos modais será representado por:

$$
I^{*}(\mathfrak{R}):=\{([x],\{E, U\}) \mid[x] \in I(\mathfrak{R})\} .
$$

Com $\mathfrak{R}$ representando o conjunto dos números reais.

As coordenadas modais de um intervalo modal são seu intervalo clássico (ext) e sua modalidade $(\bmod )$. Especificamente, dado $[x]^{\prime}=\left([x], \mathrm{Q}[x]^{\prime}\right) \in \mathrm{I}^{*}$ ( $\mathfrak{R})$, então:

$$
\begin{gathered}
\operatorname{ext}\left([x], Q[x]^{\prime}\right):=[x] \\
\bmod \left([x], Q[x]^{\prime}\right):=Q[\mathrm{x}]^{\prime}
\end{gathered}
$$


Um intervalo modal é dito intervalo próprio (e existencial), se o extremo inferior é menor que o extremo superior, caso contrário é dito intervalo impróprio (e universal).

Utilizando a notação canônica, a operação do quantificador $Q$, pode ser visualizada a seguir:

$$
Q\left(x,[a, b]^{\prime}\right)=\left\{\begin{array}{l}
\text { se } a \leq b \text { então } E(x,[a, b]), \\
\text { se } a \geq b \text { então } U(x,[b, a]),
\end{array}\right\}
$$

As operações aritméticas para intervalos modais são semelhantes às dos intervalos clássicos, estas são definidas por:

$$
\begin{aligned}
& {[a]^{\prime}+[b]^{\prime}=\left[a^{-}+b^{-}, a^{+}+b^{+}\right]^{\prime},} \\
& {[a]^{\prime}-[b]^{\prime}=\left[a^{-}-b^{+}, a^{+}-b^{-}\right]^{\prime},}
\end{aligned}
$$

$[a]^{\prime} \cdot[b]^{\prime}=\left[a^{-} b^{-}, a^{+} b^{+}\right]^{\prime}$ se $a^{-} \geq 0, a^{+} \geq 0, b^{-} \geq 0, b^{+} \geq 0$,

$$
\begin{array}{r}
\alpha \cdot[a]^{\prime}=\left[\alpha a^{-}, \alpha a^{+}\right]^{\prime}, \text { se } \alpha \geq 0, \\
=\left[\alpha a^{+}, \alpha a^{-}\right]^{\prime}, \text { se } \alpha \leq 0, \\
{[a]^{\prime} /[b]^{\prime}=\left[a^{-} / b^{+}, a^{+} / b^{-}\right],} \\
\text {se } a^{-} \geq 0, a^{+} \geq 0, b^{-} \geq 0, b^{+} \geq 0,
\end{array}
$$

\section{Extensões Intervalares Semânticas Modais}

Dada uma função $f$ de $n$ variáveis, $x_{1}, x_{2}, \ldots, x_{n}$, contínua em $[x] \in \mathrm{I}^{*}\left(\mathfrak{R}^{n}\right)$, define-se a imagem $\operatorname{de} f$ por:

$$
R_{f}([x]):=.\left[\min _{x \in[x]} f(x), \max _{x \in[x]} f(x)\right]
$$

Muitas vezes calcular $R_{f}([x])$ representa uma tarefa complexa. Quando $f$ é uma função racional, em vez de $R_{f}([x])$, pode-se obter mais facilmente a extensão intervalar de $f$ em $[x], f_{R}([x])$, substituindo os argumentos escalares da função por argumentos intervalares e as operações reais por operações intervalares correspondentes (Prado, 2006). Sendo assim, $R_{f}([x]) \subseteq f_{R}([x])$. Portanto, o único significado para $Z:=R_{f}([\mathrm{x}])$ ou $Z:=f_{R}([x])$ é:

$$
U\left(x_{1} \in\left[x_{1}\right]\right) \ldots U\left(x_{n} \in\left[x_{n}\right]\right) E(z \in Z) z=f\left(x_{1}, . . x_{n}\right) .
$$

No contexto de intervalos modais, assim como um predicado $P(x)$ leva a um predicado intervalar $Q\left(x,[x]^{\prime}\right) P(x)$, a relação $z=f\left(x_{1}, . . x_{n}\right)$ pode levar a uma relação intervalar $Z=F\left(\left[x_{1}\right] \ldots\left[x_{n}\right]\right)$ representando um predicado da forma:

$$
\begin{gathered}
Q_{1}\left(x_{1} \in\left[x_{1}\right]\right) \ldots Q\left(x_{\mathrm{n}} \in\left[x_{\mathrm{n}}\right]\right) \\
Q_{z}\left(z \in F\left(\left[x_{1}\right] \ldots\left[x_{n}\right]\right)\right) z=f\left(x_{1}, \ldots x_{n}\right) .
\end{gathered}
$$

A função $F: I^{*}\left(\Re^{n}\right) \rightarrow I(\Re)$ é chamada de extensão semântica intervalar de $f$. A seguir são definidas duas outras extensões semânticas de $f$ (Prado, 2006).
Seja $f$ uma função contínua de $\mathfrak{R}^{\mathrm{n}}$ em $\mathfrak{R},[x]^{\prime} \in$ $I^{*}\left(\Re^{\mathrm{n}}\right)$, e $\left(x_{p}, x_{i}\right)$ componentes de separação de $[x]^{\prime}=$ ( $\left.\left[x_{p}\right]^{\prime},\left[x_{i}\right]^{\prime}\right)$, com $\left[x_{p}\right]^{\prime}$ um subvetor contendo as componentes próprias e $\left[x_{i}\right]^{\prime}$ contendo as componentes impróprias de $[x]^{\prime}$ (Prado, 2006). Definem-se as extensões intervalares semânticas modais $f^{*}$ e $f^{* *}$ pelas equações abaixo.

$$
\begin{aligned}
& f *\left([x]^{\prime}\right)=\left[\begin{array}{l}
\min _{x_{p} \in\left[x_{p}\right]}\left\{\max _{x_{i} \in\left[x_{i}\right]} f\left(x_{p}, x_{i}\right)\right\}, \\
\max _{x_{p} \in\left[x_{p}\right]}\left\{\min _{x_{i} \in\left[x_{i}\right]} f\left(x_{p}, x_{i}\right)\right\}
\end{array}\right] \\
& f * *\left([x]^{\prime}\right)=\left[\begin{array}{c}
\left.\max _{x_{p} \in\left[x_{p}\right]}\left\{\min _{x_{x_{i} \in\left[x_{i}\right]}} f\left(x_{p}, x_{i}\right)\right\},\right\} \\
\min _{x_{p} \in\left[x_{p}\right]}\left\{\max _{x_{i} \in\left[x_{i}\right]} f\left(x_{p}, x_{i}\right)\right\}
\end{array}\right]
\end{aligned}
$$

Importantes relações de inclusão entre $f^{*}\left([x]^{\prime}\right) \mathrm{e}$ $f^{* * *}\left([x]^{\prime}\right)$ são:

$$
\begin{aligned}
& f^{*}\left([x]^{\prime}\right) \subseteq f^{*} *\left([x]^{\prime}\right), \\
& {[x]^{\prime} \subseteq\left([b]^{\prime}\right.} \Rightarrow f^{*}\left([x]^{\prime}\right) \subseteq f^{*}\left([b]^{\prime}\right), \\
& f^{* *}\left([x]^{\prime}\right) \subseteq f^{* *}\left([b]^{\prime}\right)
\end{aligned}
$$

Uma função $f$ é dita unimodal em um intervalo $[x]$ ' se $f$ for totalmente monótona em todo intervalo $[x]$ ', ou seja, se $f$ for totalmente crescente ou decrescente em $[x]$ '. Quando $f$ é unimodal, as extensões são iguais. Além disso, se $[x]$ ' é um intervalo próprio, $f^{*}$ é a imagem de $f$ no domínio $[x]$ (Prado, 2006).

\section{Teoremas Semânticos}

As extensões semânticas $f^{*}$ e $f^{* *}$ estão associadas à extensão semântica $F$ e podem ser utilizadas para atribuir significados aos resultados de cálculos intervalares. Mas para isso, faz-se uso de dois teoremaschave, chamados teoremas semânticos.

Teorema 2.1 (Teorema Semântico para $\left.f^{*}\right)$. Se $[x]^{\prime} \in \mathrm{I}^{*}\left(\mathfrak{R}^{\mathrm{n}}\right), f$ é contínua em $[x]$ e $F\left([x]^{\prime}\right) \in \mathrm{I}^{*}(\mathfrak{R})$, então $f^{*}\left([x]^{\prime}\right) \subseteq F\left([x]^{\prime}\right)$ se e somente se (Gardenes et al, 2001):

$$
U\left(x_{p} \in\left[x_{p}\right]\right) Q\left(z, F\left([x]^{\prime}\right)\right) E\left(x_{i} \in\left[x_{i}\right]\right)\left(z=f\left(x_{p}, x_{i}\right)\right) .
$$

Teorema 2.2 (Teorema Semântico para $f^{* *}$ ). Se $[x]^{\prime} \in \mathrm{I}^{*}\left(\mathfrak{R}^{\mathrm{n}}\right), f$ é contínua em $[x]$ e $F\left([x]^{\prime}\right) \in \mathrm{I}^{*}(\mathfrak{R})$, então $f^{* *}\left([x]^{\prime}\right) \supseteq F\left([x]^{\prime}\right)$ se e somente se (Gardenes et al, 2001):

$$
\begin{gathered}
U\left(x_{i} \in\left[x_{i}\right]\right) Q\left(z, \operatorname{Dual}\left(F\left([x]^{\prime}\right)\right)\right) \\
E\left(x_{p} \in\left[x_{p}\right]\right)\left(z=f\left(x_{p}, x_{i}\right)\right) .
\end{gathered}
$$

Os Teoremas 2.1 e 2.2 estabelecem condições gerais para extensões $F: I^{*}\left(\Re^{\mathrm{n}}\right) \rightarrow I^{*}(\Re)$ em termos das semânticas $f^{*}\left([x]^{\prime}\right)$ e $f^{* *}\left([x]^{\prime}\right)$, mas não indicam 
como essas são calculadas. A seguir será explicitada uma indicação de como calculá-las (Prado, 2006).

\section{Extensões Racionais Modais}

O cálculo de $f^{*}$ ou $f^{* *}$ é em geral uma tarefa difícil, o procedimento usual é determinar aproximações internas de $f^{*}$ e externas de $f^{* *}$ que mantenham as interpretações semânticas dos Teoremas 2.1 e 2.2.

Seja $f$ uma função contínua racional no domínio $[x]$. A extensão definida pela sequência de operações e indicada pela sintaxe de $f$ é chamada extensão racional modal, $f_{R}\left([x]^{\prime}\right)$. Segundo Prado (2006) existem vários resultados relacionados à extensão intervalar racional modal $f^{*}$ e $f^{* *}$. Para um detalhamento sobre como obter $f_{R} *\left([x]^{\prime}\right)$ e $f_{R} * *\left([x]^{\prime}\right)$, as extensões racionais correspondentes às aproximações interna e externa de $f^{*}$ e $f^{* *}$, respectivamente ver (Gardenes et al, 2001).

\section{Metodologia}

A metodologia de trabalho utilizada na pesquisa foi, inicialmente, fazer um levantamento bibliográfico sobre o assunto teoria de controle, controle robusto e aritmética intervalar modal. Em seguida, foi necessário realizar a modelagem matemática do motor $\mathrm{CC}$. Por ultimo, foi realizado o projeto do controlador robusto utilizando aritmética intervalar modal e simular os resultados obtidos.

\subsection{Modelagem}

A modelagem do motor CC é realizada através de equações diferenciais, que representam a dinâmica do sistema (Dorf, 2001). Utilizando equações diferenciais e a transformada de Laplace, obtém-se sua função de transferência, que é dada por:

$$
\begin{gathered}
G_{p}=\frac{K_{m}}{\tau_{m} s^{2}+s} \\
K_{m}=\frac{K_{T}}{R_{a} B+K_{T} K_{E}}
\end{gathered}
$$

e

$$
\tau_{m}=\frac{R_{a} J}{R_{a} B+K_{T} K_{E}}
$$

Em que $K_{T}$ é uma constante de acoplamento e é igual a $10,9 . \times 10^{-3} \mathrm{Nm} / \mathrm{A}, R_{a}$ é a resistência de 13,6 $\Omega, B$ é o coeficiente de atrito viscoso e equivale a $8,813 \times 10-2 \mathrm{Kgm}^{2} / \mathrm{s}, K_{E}$ é a constante de força contraeletromotriz com valor igual a $1,1528 \times 10^{-4} \mathrm{Vs} / \mathrm{rad}$ e $J$ a $2,08 \times 10^{-7} \mathrm{Kgm}^{2}$. Os dados foram adquiridos através do fabricante Maxon Motor (Maxon, 2011). Substituindo estes valores, $K_{m}$ é igual 89,9927 e $\tau_{m}$ é 0,0236 .

\subsection{Controle Robusto PD com Intervalos Modais}

Através das equações (3) e (10) calcula-se a função de transferência de malha fechada, que é dada pela equação:

$$
G_{M F}=\frac{\left(K_{m} k_{p}\right) / \tau_{m}}{s^{2}+\frac{s\left(1+K_{m} k_{d}\right)}{\tau_{m}}+\left(K_{m} k_{p}\right) / \tau_{m}}
$$

Para encontrarmos os ganhos do controlador, é necessário realizar uma comparação entre o denominador de $G_{M F}$ e a equação padrão de segunda-ordem (equação característica).

$$
s^{2}+2 \xi \omega_{n} s+\omega_{n}^{2}=0
$$

Onde $\xi$ é coeficiente de viscosidade e $\omega_{n}$ é a frequência natural do sistema. Como o projeto se trata de um sistema robótico, convém que o sistema não deva ter oscilação e o mesmo tem que apresentar uma resposta rápida quando excitado por uma entrada. Portanto, serão consideradas como especificações de projeto, que a resposta não tenha oscilação e que atenda a um tempo de acomodação especificado. Sendo assim, $\xi$ tem que ser criticamente amortecido, ou seja, igual a 1 e $\omega_{n}$ tem que ser um valor suficientemente alto, definimos como $91 \mathrm{rad} / \mathrm{s}$ (e de acordo com a equação (4), o tempo de acomodação torna-se $t_{s}=0.0527 \mathrm{~s}$ ). Comparando o denominador da equação (13) com a equação (14), ajustam-se os ganhos do controlador:

$$
\begin{gathered}
k_{d}=\frac{2 \xi \omega_{n} \tau_{m}-1}{K_{m}} \\
k_{p}=\frac{\omega_{n}^{2} \tau_{m}}{K_{m}}
\end{gathered}
$$

Como citado anteriormente, neste projeto de controle robusto, levamos em consideração incertezas nos parâmetros do motor CC. Considerando os parâmetros da planta como intervalos, os mesmos foram definidos por uma variação percentual para mais e para menos em cima dos valores originais.

Em projetos de controle, há necessidade de garantir que, para qualquer valor dentro dos intervalos modais, referente à planta seja possível obter um controlador, ou seja, devemos fazer esta consideração semântica $\forall K_{m} \in\left[K_{m}\right]$ ' e $\forall \tau_{m} \in\left[\tau_{m}\right]^{\prime}$. E para especificações em termos de resposta transitória, devem ser consideradas que, $\exists \xi \in[\xi]$ 'e $\exists \omega_{n} \in\left[\omega_{n}\right]$ ', pois basta que se garanta que a especificação pertença ao intervalo aceitável de especificações. Neste caso, $\xi$ deve ser igual a 1 , sendo um intervalo pontual. Assim como os parâmetros da planta, os ganhos do controlador a serem projetados devem ter a mesma interpretação semântica, ou seja, $\forall \mathrm{k}_{\mathrm{d}} \in\left[k_{d}\right]$ ' e $\forall$ 
$k_{p} \in\left[k_{p}\right]^{\prime}$, para garantir que todos os valores do intervalo podem ser usados (Prado, 2008). Desta forma, a interpretação semântica geral, de acordo com as especificações de controle é:

$$
\begin{gathered}
\left.U\left(K_{m} \in\left[K_{m}\right]^{\prime}\right) U\left(\tau_{m} \in\left[\tau_{m}\right]^{\prime}\right)\right) E\left(\omega_{n} \in\left[\omega_{n}\right]^{\prime}\right) \\
U\left(k_{d} \in\left[k_{d}\right]^{\prime}\right) U\left(k_{p} \in\left[k_{p}\right]^{\prime}\right) .
\end{gathered}
$$

Para obter esta interpretação semântica aplicamos o primeiro teorema semântico às equações de cálculos de ganhos do controlador, temos que os intervalos dos parâmetros devem ser próprios e o intervalo da especificação, juntamente com os dos ganhos do controlador devem ser impróprios. Vale destacar que ao realizar os cálculos dos ganhos, os intervalos podem ser próprios, o que significaria que dentro dos intervalos encontrados existiria um controlador que atenderia às especificações, porém não se saberia qual é. Ainda seria necessário fazer algum tipo de busca para encontrar pelo menos um controlador que atendesse às especificações.

Para obter as extensões semânticas intervalares para o cálculo dos ganhos, faz-se uso das equações (17) e (18) e intervalos modais, resultando em:

$$
\begin{gathered}
{\left[k_{d}\right]^{\prime}=\frac{2 \xi\left[\omega_{n}\right]^{\prime}\left[\tau_{m}\right]^{\prime}-1}{\left[K_{m}\right]^{\prime}}} \\
{\left[k_{p}\right]^{\prime}=\frac{\left[\omega_{n}^{2}\right]^{\prime}\left[\tau_{m}\right]^{\prime}}{\left[K_{m}\right]^{\prime}}}
\end{gathered}
$$

Desenvolvendo as equações (17) e (18) utilizando as operações de aritmética intervalar modal (Prado, 2006) e considerando que todos os parâmetros $\left(\tau_{m}, K_{m}, \xi, \omega_{n}\right)$ são positivos, obtém-se as extensões semânticas intervalares dos ganhos como:

$$
\begin{gathered}
{\left[k_{d}\right]^{\prime}=\left[\frac{2 \xi \omega_{n}^{-} \tau_{m}^{-}-1}{K_{m}^{+}}, \frac{2 \xi \omega_{n}^{+} \tau_{m}^{+}-1}{K_{m}^{-}}\right]} \\
{\left[k_{d}\right]^{\prime}=\left[\frac{\left(\omega_{n}^{-}\right)^{2} \tau_{m}^{-}}{K_{m}^{+}}, \frac{\left(\omega_{n}^{+}\right)^{2} \tau_{m}^{+}}{K_{m}^{-}}\right]}
\end{gathered}
$$

Como as funções para os cálculos dos ganhos são unimodais, as funções intervalares semânticas modais são iguais. De acordo com Gardenes (2001), como não há multi-incidência de variáveis $f_{R}\left([x]^{\prime}\right)=$ $f_{R} *\left([x]^{\prime}\right)=f_{R} * *\left([x]^{\prime}\right)$.

\section{Resultados}

Assumindo uma variação de $10 \%$ em torno dos parâmetros originais da planta, os intervalos correspondem a: $\left[K_{m}\right]=[80,9934,98,9920]$ e $\left[\tau_{m}\right]=[0,0212$, $0,0260]$. Já para a especificação de projeto ficou: $\xi$ como valor pontual igual a 1 e $\left[\omega_{n}\right]=[110,72]$.
Percebe-se que os parâmetros da planta são intervalos próprios e da especificação, $\left(\omega_{n}\right)$, impróprio.

Através das equações (19) e (20), pode-se obter $\left[\mathrm{k}_{\mathrm{d}}\right]=[0,0371,0,0338]$ e $\left[k_{p}\right]=[2,5962,1,6616]$. É possível perceber que os intervalos são impróprios. Portanto, as semânticas referentes às equações (19) e (20) são, respectivamente:

$$
\begin{aligned}
& U\left(K_{m} \in\left[K_{m}\right]^{\prime}\right) U\left(\tau_{m} \in\left[\tau_{m}\right]^{\prime}\right) E\left(\omega_{n} \in\left[\omega_{n}\right]^{\prime}\right) \\
& U\left(k_{d} \in\left[k_{d}\right]^{\prime}\right) k_{d}=\frac{2 \xi \omega_{n} \tau_{m}-1}{K_{m}}
\end{aligned}
$$

e

$U\left(K_{m} \in\left[K_{m}\right]^{\prime}\right) U\left(\tau_{m} \in\left[\tau_{m}\right]^{\prime}\right) E\left(\omega_{n} \in\left[\omega_{n}\right]^{\prime}\right) U\left(k_{p} \in\left[k_{p}\right]^{\prime}\right) k_{p}=\frac{\tau_{m} \omega_{m}^{2}}{K_{m}}$

Ou seja, para quaisquer valores de $k_{d}$ e $\mathrm{k}_{\mathrm{p}}$ dentro dos seus intervalos, existirão valores de dentro do intervalo da especificação para quaisquer valores $K_{m}$ e $\tau_{m}$ dentro dos seus limites de $10 \%$ do valor central que foi obtido no modelo.

Na Figura 3 são apresentadas as respostas ao degrau unitário dos sistemas controlados, considerando diversas variações para a planta a partir de várias combinações dos parâmetros incertos (dentro do intervalo utilizado no projeto, incluindo os valores extremos), e também diversas combinações de valores de ganhos para o controlador (dentro do intervalo dos resultados dos ganhos, incluindo os valores extremos). As respostas mais rápidas foram obtidas com os maiores valores de ganhos dos intervalos, e as mais lentas, com os menores valores. De acordo com a Figura 3, todas as respostas tiveram tempo de acomodação $\left(t_{s}\right)$ atendendo à especificação de $0,0667 \mathrm{~s}$, utilizando o valor mínimo de $\omega_{n}$ de 72 $\mathrm{rad} / \mathrm{s}$. Além disso, o sistema não apresentou sobressinal, que é a especificação de $\xi$ igual a 1, conforme esperado pelo projeto. O melhor valor para os controladores dentro de seus respectivos intervalos são os valores máximos $\left(k_{d}=0,0371\right.$ e $\left.k_{p}=2,5962\right)$, pois a velocidade de resposta é maior.

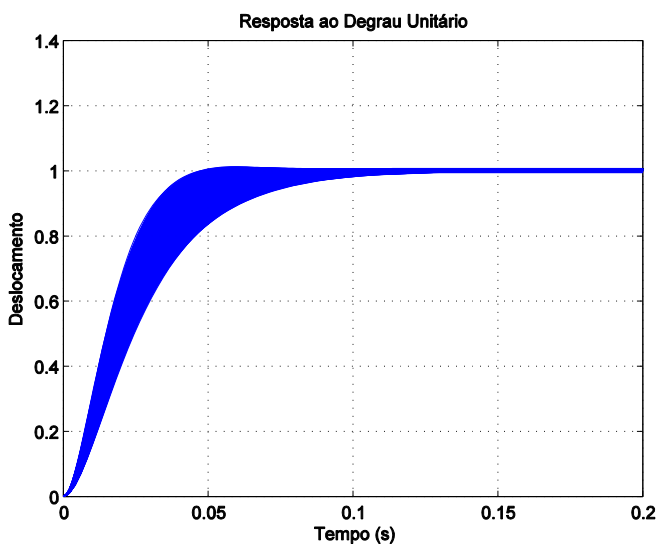

Figura 3 - Respostas do sistema com o PD robusto.

Além disso, foram realizadas simulações no $\mathrm{Si}$ mulink considerando vários tipos de valores para a 
referência e visualizado o sinal de controle. O sistema foi simulado levando em conta que o mesmo está sendo implementado utilizando o controlador embarcado em microcontrolador. Portanto, todos os sinais já são considerados digitalizados e variando de 0 a 1023. No caso da referência, os ângulos variam de 0 a 270 graus. Os gráficos de uma simulação utilizando os valores máximos de ganho são apresentados nas Figuras 4 e 5, mostrando o sinal de saída e referência (ângulos de aproximadamente 30 e 60 graus) e o sinal de controle (tensão), respectivamente.

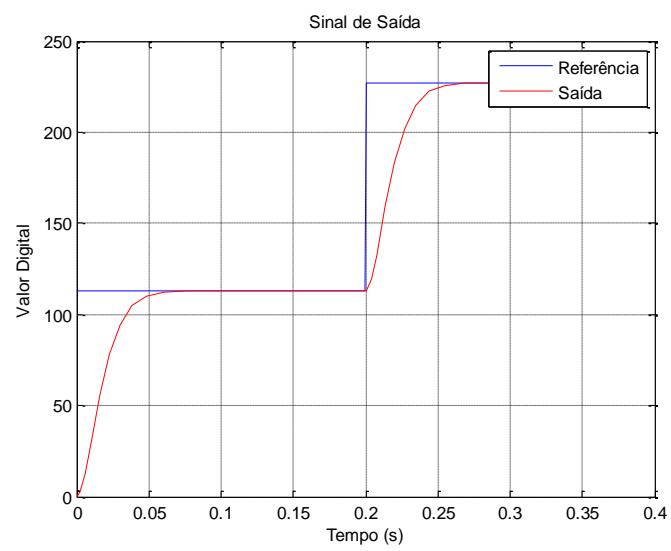

Figura 4 - Sinal de Saída

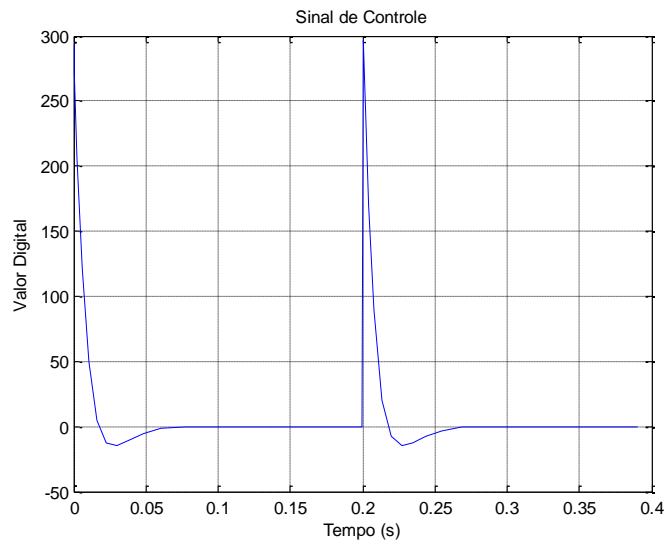

Figura 5 - Sinal de Controle

\section{Conclusão}

Neste artigo foi proposta uma abordagem para um projeto de controlador robusto utilizando a estrutura PD por realimentação de velocidade juntamente com análise intervalar modal para um motor CC. Através do resultado demonstrado na Figura 3, percebe-se que mesmo o sistema sujeito a incertezas nos parâmetros da planta, as respostas do sistema controlado, usando diversos valores de ganhos dentro do intervalo obtido, se mantêm dentro das especificações de projeto. Portanto, o uso de análise intervalar modal em projetos de controladores robustos para um motor CC de uma junta de um manipulador robótico, se mostrou bastante satisfatória. Pretende-se ainda realizar testes com perturbações nas simulações, e este sistema com o controlador está em fase de im- plementação prática. Vale ressaltar que para esta mesma planta, foi realizado o projeto utilizando análise intervalar clássica via programação alvo (Lordelo, 2004), porém os resultados não foram satisfatórios, mas os detalhes encontram-se em (Santos, 2013).

\section{Agradecimentos}

Este trabalho teve o apoio do CNPq e da UEFS.

\section{Referências Bibliográficas}

Ackermann, J. (1993). Robust control:systems with uncertain Physical Parametrs, Springer-Verlag, New York, NY. DOI: 10.1007/978-1-4471-3365-0

Barmish, B. R. (1994). New tolls for robustness of linear systems, Macmillan Publishing Co. New York, NY.

Bhattacharyya, S. P; Chapellat, H; Keel, L. H. (1995). Robust control:the parametric approach, Prentice-Hall, Upper Saddle River, NJ.

Dorf, R.C; Bishop, R.H. (2001). Sistemas de controle modernos, LTC.

Gardenes, E. SAINZ, M; Jorba, L.; Calm, R; Estela, R; Mielgo, H. and Trepat, A. (2001). Modal Intervals, reliablecomputing 7:77-111.

Gutierrez, J. (2010). Usando técnicas avançadas de controle industrial, NationalInstruments. Disponível

em: <http://digital.ni.com/worldwide/brazil.nsf/web/ all/3E2C24128C0B08C78625757F006782FA> Acesso: em 05 jul. 2011.

Lordelo, A. D. S. (2004). Análise e projeto de controladores robustos por alocação de pólos via análise intervalar. Campinas, SP, 2004. Origininalmente apresentada como dissertação de doutorado, Universidade Estadual de Campinas.

Motor Maxon. (2011). Disponível em: <http://www.maxonmotor.com/> Acesso: em 3 maio. 2011.

Ogata, K. (2003). Engenharia de controle moderno, Prentice Hall.

Prado, M. L. P. (2006). Controle robusto por alocação por pólos via análise intervalar modal. Dissertação (Doutorado em Engenharia Eletrica)Universidade Estadual de Campinas, Campinas.

Prado, M. L. P. e Paz, M. A. (2008). Controle robusto utilizando estrutura PD e análise intervalar modal. In: XIII Congresso Latinoamericano de Control Automatico, Mérida.

Santos, V. A. B. dos. (2013). Controle Robusto por Alocação de Pólos para um Motor de uma Junta Robótica. Monografia de Trabalho de Conclusão de Curso. Universidade Estadual de Feira de Santana, Feira de Santana.

Zhou, K.; Doyle, J. C.; Glover, K. (1996). Robust and Optimal Control. 1. ed. Upper Saddle River, New Jersey: Prentice Hall. DOI: $10.1137 / \mathrm{S} 0363012993252711$ 